\section{anthropology} \& materialism

\section{Anthropology \& Materialism}

A Journal of Social Research

1 | 2013

Across the Fields

\title{
Dans les champs magnétiques du matérialisme et de l'anthropologie
}

In the Magnetic Fields of Materialism and Anthropology

En los campos magnéticos del materialismo y de la antropología

\section{Marc Berdet}

\section{(2) OpenEdition}

\section{Journals}

Édition électronique

URL : http://journals.openedition.org/am/139

DOI : 10.4000/am.139

ISSN : 2364-0480

Éditeur :

CETCOPRA, CRASSH - Center for Research in the Arts Social Sciences and Humanities, Fakultät Gestaltung - Universität der Künste Berlin

Référence électronique

Marc Berdet, « Dans les champs magnétiques du matérialisme et de l'anthropologie », Anthropology \& Materialism [En ligne], 1 | 2013, mis en ligne le 15 octobre 2013, consulté le 22 septembre 2020. URL : http://journals.openedition.org/am/139; DOI : https://doi.org/10.4000/am.139

Ce document a été généré automatiquement le 22 septembre 2020

Tous droits réservés 


\title{
Dans les champs magnétiques du matérialisme et de l'anthropologie
}

\author{
In the Magnetic Fields of Materialism and Anthropology \\ En los campos magnéticos del materialismo y de la antropología
}

\section{Marc Berdet}

«Car, en dépit de l'accord le plus fondamental et le plus concret par ailleurs, tous les points sur lesquels je diffère de vous pourraient se résumer sous le titre d'un matérialisme anthropologique, que je ne peux suivre.

C'est comme si pour vous le corps humain était la mesure de la concrétude. »

« L'induction immédiate de l'impôt sur le vin à l'

Âme $d u$ vin [le poème de Baudelaire] attribue justement aux phénomènes cette sorte de spontanéité, d'évidence concrète, d'épaisseur qu'ils ont perdue dans le capitalisme. Cette sorte de matérialisme immédiat, anthropologique, j'aimerais presque le répéter, cache un élément profondément romantique [...] Pour parler très drastiquement, on pourrait dire que votre travail se situe au carrefour de la magie et du positivisme. Cet endroit est ensorcelé. »

Theodor Adorno à Walter Benjamin, 6 sept. 1936

et 10 nov. 1938

1 Nous voici, donc, au croisement de l'anthropologie et du matérialisme. Le lecteur s'interrogera d'emblée : que se passe-t-il quand l'anthropologie se fait matérialiste ? Et lorsque le matérialisme devient anthropologique? L'expression lance-t-elle un oxymore de plus sur le marché aux slogans équivoques? Non. Elle génère une polarisation précise qui crée un champ magnétique dans l'inconfort duquel nous 
souhaiterions nous installer. Elle conduit, dans une direction, à une anthropologie matérialiste et, dans l'autre, à un matérialisme anthropologique tel qu'il a été esquissé par Walter Benjamin ${ }^{1}$.

2 Ce numéro se divise en quatre sections. La première section comprend des articles théoriques qui portent sur le matérialisme de différents représentants de la théorie critique (1. Théorie sociale). La deuxième section rassemble une pièce radiophonique de Walter Benjamin, une interprétation de sa relation aux nouveaux médias et au jeu d'enfant, ainsi que les analyses d'un monument berlinois et des fantasmagories modernes (2. Expérimentations matérialistes). La troisième section contient une discussion des conceptions traditionnellement critiques de l'Etat (3. Polémiques), et la quatrième une recension d'un ouvrage collectif sur Charles Fourier et Walter Benjamin (4. Recensions).

\section{Sept remarques préliminaires}

3 Nous nous consacrons particulièrement au matérialisme anthropologique dans le présent numéro. Il serait déplacé de tenter ici une définition positive et déterminée de ce terme. Il semble utile cependant, à titre expérimental, d'en tenter une brève formalisation. Ces sept remarques visent donc à circonscrire le matérialisme anthropologique en tant que principe actif dans la pensée de Walter Benjamin tel qu'il fait sens pour nous aujourd'hui².

UNE TRADITION SINGULIÈRE. Chez Walter Benjamin, le matérialisme anthropologique désigne rétrospectivement un groupe d'auteurs très disparates, du XIX ${ }^{e}$ siècle et des années 1930, de langue française ou allemande. Ces auteurs ne forment pas de groupe autoproclamé, ne se rapportent pas explicitement les uns aux autres et ne se revendiquent pas toujours d'un type de matérialisme particulier.

TERME POLÉMIQUE. Le matérialisme anthropologique ne se confond stricto sensu ni avec le socialisme scientifique, ni avec un quelconque marxisme romantique, ni avec aucune sorte d'humanisme (et cela bien qu'il contienne des éléments scientifiques, romantiques ou humanistes) ${ }^{3}$.

UNE SENSIBILITÉ. Le matérialisme anthropologique est une méthode ou, pour le dire plus précisément, une sensibilité. Celle-ci s'avère capable de saisir un moment ludique au sein de l'action et une intensité poétique dans l'histoire que les filets du matérialisme traditionnel sont incapables de capturer. Ce matérialisme poétique agit comme une métonymie qui indiquerait un noyau historique impossible à symboliser sans en dissoudre aussitôt la spécificité.

UNE TEMPORALITÉ. Le matérialisme anthropologique incarne une temporalité verticale qui interrompt à la fois la temporalité horizontale de la marche victorieuse des "héros » conquérants, et la temporalité cyclique de l'industrie productrice de marchandises.

UN CONCEPT. Le matérialisme anthropologique est un concept qui permet de "corriger » le matérialisme dialectique. Il nous permet certes de comprendre Walter Benjamin à nouveau frais mais, surtout, il indique un work in progress chez Benjamin lui-même qui exige de nous que l'on actualise sa méthode.

UN CONCEPT INTERNE. Le matérialisme historique inclut le matérialisme anthropologique, bien que le matérialisme historique n'en ait pas pleinement conscience. Le matérialisme anthropologique doit être délibérément «intégré » (et non pas 
désintégré) dans le matérialisme historique. Autrement dit, il doit se maintenir au cœur du matérialisme historique comme un élément qui lui reste étranger.

UNE ACTUALITÉ. Lié à l'analyse dialectique du capital, le matérialisme anthropologique nous aide à comprendre notre présent. Il nous permet de localiser les forces de l'ivresse clôturées dans un imaginaire collectif soumis au fétichisme de la valeur marchande, que l'on nommera fantasmagorie ${ }^{4}$.

\section{Matérialisme anthropologique}

11 Trois contributions vont dans le prolongement de ces remarques. L'article inaugural de Marc Berdet tente une première définition positive du matérialisme anthropologique : rien à voir, ou si peu, avec le matérialisme de Feuerbach ${ }^{5}$. Il s'agit d'une étrange «tradition» évoquée par Walter Benjamin au gré d'une énumération rhapsodique d'écrivains hétérogènes. Berdet en fait ressortir cinq grandes «familles » et, tirant un fil de l'œuvre du philosophe, dégage ce qui pourrait en former un principe commun : la traduction, dans le monde humain, des forces d'attraction et de répulsion du monde de la nature. Déplaçant le cadre conceptuel du matérialisme dialectique, le matérialisme anthropologique consisterait en la traduction bizarre et poétique de Newton en Jean Paul, Büchner, Fourier, Balzac et Rimbaud (Un matérialisme "stupéfiant». Entre matérialisme anthropologique et matérialisme dialectique).

«Rimbaud avec Marx »: Michaël Löwy reprend à son compte ce mot d'ordre d'André Breton pour interpréter le matérialisme anthropologique des surréalistes. Le matérialisme anthropologique participe à un romantisme anticapitaliste que le marxisme se doit d'intégrer s'il veut être réellement critique, et ne pas abandonner l'utopie ${ }^{6}$. Le mouvement surréaliste incarne en ce sens une rébellion contre le désenchantement et la rationalité instrumentale propres à la civilisation capitaliste. Matérialisme anthropologique et matérialisme dialectique opèrent ainsi un alliage au sens alchimique du terme. Les non moins alchimiques «illuminations profanes»du surréalisme, intégrées au projet révolutionnaire, en forment l'exemple paradigmatique (Walter Benjamin et le surréalisme. Les noces chimiques des deux matérialismes).

13 «Fourier avec Marx »: le matérialisme anthropologique, c'est aussi la manière dont Walter Benjamin accorde l'harmonie des passions humaines avec le mouvement révolutionnaire, le phalanstère avec le Manifeste. La recension par Carlos Perez d'un Cahier Fourier spécialement consacré aux relations entre Fourier et Benjamin suggère de nombreuses pistes permettant d'articuler, de manière critique, ce matérialisme poétique avec la tradition marxienne.

14 Chacun de ces textes, de manière très différente, donne une idée de ce que peut être le matérialisme anthropologique. Mais le croisement du matérialisme et de l'anthropologie ne se limite pas à cela. Il génère aussi, sous le signe d'une théorie critique élargie, un nouveau type d'anthropologie, que l'on peut nommer « anthropologie matérialiste ».

\section{Anthropologie matérialiste}

C'est du point de vue de quatre auteurs appartenant à cette théorie critique que l'article de Dennis Johannssen s'interroge sur cette rencontre. La nécessité matérialiste 
d'historiciser tout fait humain conduit Max Horkheimer à condamner l'anthropologie allemande de son époque, aujourd'hui de nouveau sur le devant de la scène. Une anthropologie positive lui semble impossible, et même condamnable. Pour Theodor Adorno, le simple usage du terme d'«homme " est démagogique, et l'ethnologie et l'anthropologie culturelle qui prétendent à une définition "positive " de l'homme sombrent bien souvent dans le positivisme tout court. Ulrich Sonnemann, l'auteur trop peu connu d'une Anthropologie négative, tenta de réaliser le programme d'une anthropologie dialectique et matérialiste qu'Adorno appelait de ses vœux. Renoncer, y compris dans la conception marxiste, à une conception positive de l'homme, constitue le seul moyen d'en déployer toutes les possibilités dans l'histoire - et sur le sentier de l'utopie. L'anthropologie critico-utopique de Walter Benjamin fournit dans cette perspective des outils pour comprendre notre présent. Concurrente de l'anthropologie philosophique d'aujourd'hui, sa théologie n'est au fond rien qu'une stratégie matérialiste pour diagnostiquer notre époque (The Constellation of Negative Anthropology: between Critical Theory and Philosophical Anthropology).

Une théologie matérialiste : ce n'est pas le moindre des paradoxes que relève Sami Khatib dans son texte. Il existe en effet au sein des relations humaines un «faible pouvoir messianique » qui permettrait d'interrompre le cycle de l'oppression. Le messianisme de Benjamin s'avère être moins une notion théologique qu'une opération conceptuelle permettant de localiser, dans le monde profane, un pouvoir susceptible de provoquer un arrêt dans le déroulement catastrophique de l'histoire. Transformer, au sein des relations sociales capitalistes, une petite déviation en une grande : tel est le dessein qui oriente le matérialisme du philosophe allemand (The Messianic Without Messianism: Walter Benjamin's Materialist Theology).

17 Faire exploser, donc, la temporalité homogène et vide de l'histoire des vainqueurs. Certes, mais «tu ne peux pas faire exploser une relation sociale!» Ce paradoxe est le point de départ anarchiste de la polémique de Christos Lynteris. Celle-ci est dirigée contre l'Etat non pas comme "monstre froid " extérieur à l'individu, mais comme relation sociale que ce même individu actualise à chaque instant. Inspirée par Gustav Laudauer sur le terrain explicite d'une anthropologie matérialiste voire libertaire, cette polémique semble traduire politiquement la théologie matérialiste de Walter Benjamin (The State as a Social Relation).

\section{L'arc des expérimentations matérialistes}

Matérialisme anthropologique et anthropologie matérialiste forment un arc dans la tension duquel nous déployons six "expérimentations matérialistes ", tentatives de faire sortir la théorie hors de soi pour la communiquer au monde et pour que le monde y laisse son empreinte.

19 Ces six expériences matérialistes s'organisent selon trois moments : "Expérimentation matérialiste $n^{\circ} 1$ : Walter Benjamin et la radio »; «Expérimentation matérialiste $n^{\circ} 2$ : Les fantasmagories hier et aujourd'hui »; "Expérimentation matérialiste $n^{\circ} 3$ : Walter Benjamin à Berlin aujourd'hui ». Ces trois parties composent différents temps d'exploration des terres découvertes au croisement du matérialisme anthropologique et de l'anthropologie matérialiste. 
20 Pédagogue matérialiste par excellence, Walter Benjamin lui-même cherchait à transmettre quelque expérience, par ses essais radiophoniques, aux enfants. Sa pièce "Chahut autour de Kasperl», que nous republions ici, témoigne de cette tentative ludique et instructive tout en même temps. Car non seulement Walter Benjamin a écrit cette pièce, mais il l'a aussi interprétée sur les ondes, jouant avec son timbre et son articulation si significative pour se mettre dans la peau du turbulent petit Kasperl (Radau um Kasperl).

21 C'est à Philippe Baudouin que nous devons cette découverte. Dans son texte, il décrit comment il a trouvé, au cours de ses recherches, une voix qui lui semblait être celle de Walter Benjamin, et comment cela lui fut confirmé par Stéphane Hessel, dernier homme vivant sur cette terre a avoir connu le philosophe. Lui-même créateur radiophonique, Baudouin retrace le parcours de cet homme de radio que fut Walter Benjamin : parcours philosophique d'un penseur qui prenait la technique au sérieux, et en explorait les possibilités; parcours anthropologique d'un matérialiste qui prenait l'enfance au sérieux, et en exploitait les potentialités ('Give to Listener what they Want and Even a little Bit more': A Philosopher on Air).

Stefano Marchesoni prolonge en quelque sorte ce geste de Walter Benjamin radiographié par Baudouin. Lumières pour enfants, c'était le titre d'un recueil de contes radiophoniques du philosophe; Walter Benjamin pour enfants, aurait pu s'intituler la causerie que Marchesoni adressa à des enfants de 9 à 11 ans. Nous la reproduisons ici. Elle s'intitule en réalité « Que peut un enfant? Le Toy Story de Walter Benjamin ». Petite expérience matérialiste cherchant à expliquer Walter Benjamin par l'univers du jeu, la causerie de Marchesoni pénètre l'imaginaire commun des enfants pour les mettre sur les traces du petit Kasperl : ce qu'il peut vraiment, un enfant l'esquisse à chaque instant par un jeu mimétique avec les forces de ce monde (Was kann ein Kind? Walter Benjamins Toy Story).

23 Stefano Marchesoni et Meike Schmidt-Gleim éclairent les adultes là-dessus. Leur texte met le pouvoir mimétique en rapport avec une puissance créatrice qui, comme la «faible force messianique ", est susceptible de rompre le cycle de l'exploitation et de la répression. L'enfant se met dans la peau du docteur, mais aussi dans celle du train : il existe ainsi dans tout jeu enfantin un moment d'imitation du monde qui est à proprement parler le moment poétique de la créativité humaine (Bemerkungen über Mimetismus und Kindheit).

24 Le rétablissement d'une intensité poétique dans la création humaine doit cependant se faire du point de vue de la classe opprimée et combattante qui garde, en elle, le souvenir des générations passées. C'est la variante d'une des huit thèses que Marc Berdet présente sur la fantasmagorie, imaginaire bourgeois qui se concrétise dans l'environnement architectural et urbain pour y enfermer les passions fouriéristes potentiellement subversives. Ce texte entend reprendre aujourd'hui l'analyse des passages parisiens entamée par Walter Benjamin il y a presque cent ans (Eight Theses on Phantasmagoria).

Dans un dialogue expérimental, Christos Lynteris répond à ces huit thèses par dix brefs commentaires qui mobilisent l'anthropologie et la psychanalyse contemporaines. La répétition obsessive, sur le mode mythique, de la fantasmagorie, révèle alors l'enchaînement profond de cette créativité passionnelle à l'inertie de la pulsion de mort (Ten Comments on 'Eight Theses'). 

contraire ce vertige poétique dans l'activité du chercheur. L'« expérimentation matérialiste » de Joanna Kusiak épouse ainsi celle de Benjamin. Il s'agit d'une saisie des matériaux hétéroclites qui gravitent autour d'un même phénomène pour en cristalliser : a) le déploiement historique, b) la polarisation politique et c) l'intensité poétique. Ce phénomène empirique, c'est la statue massive et monumentale d'Ernst Thälmann, au nord de Berlin, dans le quartier de Prenzlauerberg. Ce monument socialiste, que la ville n'a pas pu démolir faute d'argent, reste à l'écart des circuits touristiques: ni les badauds du tourisme planétaire, ni les «alternatifs» ne s'y aventurent, et la politique de la capitale "pauvre mais sexy» est bien en mal de l'intégrer à son entreprise de séduction. Ce monument semble attirer à lui comme un trou noir les usages que la ville voudrait expulser, notamment, par-delà l'histoire récente, les rituels de nettoyage des nostalgiques du communisme et le vandalisme des néo-nazis. A partir de cet exemple, Kusiak s'affronte au matérialisme de Walter Benjamin le long d'un questionnement particulièrement pertinent : où s'arrêter dans la collecte des matériaux? Quand saisir l'intensité poétique qui en surgit ? Comment en polariser les moments pour en faire une « image dialectique » (Striking Dialectical Sparks from the Stones of Our Cities: Learning from Ernst-Thälmann-Monument in Berlin with Benjamin's Materialist Methodology)?

\section{La clé des champs}

Matérialisme anthropologique et anthropologie matérialiste ne s'arrêtent pas là pour autant. Quels sont les mythes modernes dont nous nous entourons encore aujourd'hui? Le prochain numéro sera le lieu où nous débattrons de cette question. Les numéros suivants porteront sur la technique, l'utopie et l'idéologie, avec l'espoir que le champ ouvert par la revue génère des hypothèses inédites sur chacune de ces thématiques

La revue inaugure un croisement fécond du matérialisme et de l'anthropologie qui peut prendre des chemins méthodologiques dégagés par d'autres auteurs encore, dont nous héritons. Nous pensons notamment aux travaux de Michel Foucault, véritable anthropologie construite à partir des rapports de forces entre êtres humains. La notion de biopolitique, par exemple, permet l'analyse de la capture des forces biologiques par le pouvoir?. Le "matérialisme de la rencontre" ou "aléatoire» du dernier Louis Althusser va dans le sens d'un matérialisme poétique tel que nous l'entendons ici, et cela d'autant plus qu'il devait permettre l'analyse de la stratégie de la bourgeoisie comme "règne magique et hallucinant $d u$ divertissement $"^{8}$. Des anthropologies politiques comme celles de Pierre Clastres, qui critiquait l'économisme marxiste, ou comme celle de Michaël Taussig ${ }^{9}$, qui reprend certaines thèses de Benjamin, pourraient elles aussi être au nombre d'une anthropologie matérialiste. Le matérialisme historique se limitait en effet trop souvent à la seule contradiction économique. Sa confrontation avec l'anthropologie l'ouvre à la richesse des contradictions humaines au niveau individuel des physionomies sociales aussi bien qu'au niveau collectif de l'organisation spatiale, sans parler de l'architecture, des nouvelles technologies ou des jeux de l'enfance.

En somme, la rencontre du matérialisme et de l'anthropologie pourrait jouer, par-delà les fermetures disciplinaires, le rôle de révélateur, au sens photographique du terme, de nouvelles recherches. Placées dans le giron d'une analyse critique de la domination, 
elles garderaient un œil attentif sur les magnétismes rebelles, potentiellement émancipateurs. Espérons donc que ce croisement ait de belles étincelles devant lui, et qu'il aide à ouvrir l'horizon des possibles quelque peu bouché par notre époque.

\section{BIBLIOGRAPHIE}

Abensour Miguel, L'Utopie de Thomas More à Walter Benjamin, Paris, Sens et tonka, 2000

Althusser Louis, Sur la philosophie, Paris, Gallimard, 1994

Benjamin Walter, Briefwechsel Adorno-Benjamin 1928-1940, Francfort, Suhrkamp, 1994

Benjamin Walter, Correspondance Theodor Adorno-Walter Benjamin, Paris, La Fabrique, 2003

Benjamin Walter, CEuvres, Paris, Gallimard, 2000

Benjamin Walter, Gesammelte Schriften, Francfort, Suhrkamp, 1974

Benjamin Walter, Paris, capitale du XIX ${ }^{e}$ siècle. Le livre des passages, Paris, Editions du Cerf, 1989

Clastres Pierre, Archéologie de la violence, Paris, Aube, 2010

Clastres Pierre, La société contre l'Etat, Paris, Minuit, 1974

Foucault Michel, Dits et écrits, Paris, Gallimard, 2001

Foucault Michel, Naissance de la biopolitique. Cours au Collège de France 1978-1979, Paris, Gallimard, 2004

Marx Karl, L'idéologie allemande, Paris, Editions sociales, 1982

Taussig Michael, Mimesis and Alterity. A Particular History of the Senses, Routledge, Londres, 1993

Taussig Michael, What Color is the Sacred ?, Chicago, University of Chicago Press, 2009

Sites Internet :

http://anthropologicalmaterialism.hypotheses.org/

http://biopolitica.cl/ingles/pags/portada.html

\section{NOTES}

1. Notamment dans son essai sur le surréalisme (1929) et dans son grand ouvrage inachevé, Paris, capitale du XIX $X^{e}$ siècle. Le livre des passages (1927-1940). Walter Benjamin, Euvres II, Paris, Gallimard, 2000 et Walter Benjamin, Paris, capitale du XIX $x^{e}$ siècle. Le livre des passages, Paris, Editions du Cerf, 1989. On le trouve cependant aussi ailleurs. Voir le travail engagé sur www.anthropologicalmaterialism.hypotheses.org.

2. J'ai publié une première version de ces sept remarques sur le site Internet précédemment cité. Je remercie ici Sami Khatib et Jan Sieber pour leurs commentaires à son propos, ainsi que Carlos Perez et Sébastien Broca pour leurs suggestions sur l'introduction en son entier. 
3. L'expression "matérialisme anthropologique» surgit d'ailleurs sous la plume d'Adorno lors d'une polémique épistolaire avec Walter Benjamin. Voir nos citations en exergue qui correspondent aux lettres d'Adorno du 6 septembre 1936 et du 10 novembre 1938, Walter Benjamin, Correspondance Theodor Adorno-Walter Benjamin, Paris, La Fabrique, 2003, p. 205 et 361 (Walter Benjamin, Briefwechsel Adorno-Benjamin 1928-1940, Francfort, Suhrkamp, 1994, p. 368 et Walter Benjamin, Gesammelte Schriften VII, Francfort, Suhrkamp, 1974, p. 834).

4. Voir à ce propos les « Huit thèses sur la fantasmagorie » ci-dessous.

5. Feuerbach mettait les choses à la place des mots : il arrachait les phénomènes au royaume des idées hégéliennes pour les planter dans le monde naturel. Ce matérialisme encore anhistorique aurait été « historicisé » puis «dialectisé » par Marx et Engels, ouvrant une voie royale mais problématique au socialisme scientifique, à la sociologie, à l'anthropologie, à l'histoire et à la philosophie sociales. Voir la critique classique de Feuerbach par Marx dans Karl Marx, L'idéologie allemande, Paris, Editions sociales, 1982.

6. Notamment selon les perspectives ouvertes par Miguel Abensour, par exemple dans Miguel Abensour, L'Utopie de Thomas More à Walter Benjamin, Paris, Sens et tonka, 2000.

7. Voir par exemple Michel Foucault, Dits et écrits 2, Paris, Gallimard, 2001 et Michel Foucault, Naissance de la biopolitique. Cours au Collège de France 1978-1979, Paris, Gallimard, 2004. Un réseau international s'est développé, à partir de Santiago, autour de cette problématique, visible sur http://biopolitica.cl/ingles/pags/portada.html

8. Louis Althusser, Sur la philosophie, Paris, Gallimard, 1994, p. 25.

9. Pierre Clastres a en effet montré la manière dont s'organisait matériellement, au sein d'un groupe, l'impossibilité de l'émergence d'un pouvoir hiérarchique et d'un chef d'Etat. Voir son classique Pierre Clastres, La société contre l'Etat, Paris, Minuit, 1974, mais aussi Pierre Clastres, Archéologie de la violence, Paris, Aube, 2010. Taussig s'inspire de Benjamin dans Michael Taussig, Mimesis and Alterity. A Particular History of the Senses, Routledge, Londres, 1993, ou encore plus récemment dans Michael Taussig, What Color is the Sacred ?, Chicago, University of Chicago Press, 2009.

\section{AUTEUR}

\section{MARC BERDET}

Docteur en sociologie à l'Université de la Sorbonne, postdoctorant Marie-Curie (UE) à la faculté de philosophie de l'Université de Potsdam 